\title{
Ein webbasiertes Evaluationssystem für Hochschulen
}

\author{
Ralf Vandenhouten, Miriam Selz
}

\section{Einleitung}

Angetrieben durch die Hochschulgesetzgebung, Maßnahmen zur systematischen und regelmäßigen Veranstaltungsevaluation von Fachbereichen zu ergreifen [4] [5], wurde das Projekt „Webbasiertes Evaluationssystem“ vom Total Quality Management (TQM) der Technischen Fachhochschule Wildau (TFH Wildau) angeregt. Um einen hohen Qualitätsstandard der Lehre sicherzustellen, wollte die TFH Wildau die Qualität der Veranstaltungen messbar machen und suchte nach technischen Verbesserungsmöglichkeiten.

Ziel bei diesem Projekt war eine umfassende Lösung, die es auf einfache und kostengünstige Art ermöglicht, Evaluationsdaten zu erheben, elektronisch zu erfassen und auszuwerten sowie den Dozenten und der Hochschulleitung zur Verfügung zu stellen.

Die Lehreinheiten erhalten auf diese Weise einen schnellen und fundierten Einblick in ihre Leistungen sowie eine entsprechende Einschätzung und können daraus konkrete Anregungen zur Weiterentwicklung ihrer fachlichen Kompetenz gewinnen. Die aus der Evaluierung hervorgehenden Informationen können zudem zur internen Rechenschaftslegung sowie zur kontinuierlichen Qualitätssicherung und -verbesserung genutzt werden.

\section{Hintergrund}

2.1 Das Qualitätsmanagement an der TFH Wildau In dem Zeitraum 2004-2006 wird ein umfassendes Qualitätsmanagementsystem, das TQM-Projekt (TQM: Total Quality Management oder Umfassendes Qualitätsmanagement) an der TFH Wildau eingeführt. In diesem Rahmen wurde ein hochschulspezifisches System zur internen Evaluation des Studiums an der TFH Wildau entwickelt.

Für die Regelung inhaltlicher und organisatorischer Rahmenbedingungen der internen Evaluation wurde eine zentrale Evaluationskommission unter Leitung des Vizepräsidenten Qualitätssicherung gebildet, welcher mittlerweile als fester Bestandteil in der Aufbauorganisation der TFH Wildau verankert ist. Das interne Evaluationssystem umfasst die Evaluationsarten:

- studentische Lehrevaluation

- Dozentenbefragung

- Kennzahlenbewertung

- Fachevaluation. [3]

\subsection{Evaluierung}

Die Evaluierung ist ein periodisch/zyklisch und/oder anlassbezogen genutztes Verfahren der Qualitätssicherung zur Analyse, Bewertung und Förderung der Qualität der Lehre im Hochschulwesen. Neben hochschulinternen Qualitätslenkungsmechanismen (Korrektur- und Verbes- serungsmaßnahmen) bietet sie damit Vorraussetzungen für den Leistungsvergleich mit anderen Hochschulen und kann (soll) bei der leistungsorientierten Mittelzuweisung (hochschulintern und -extern) berücksichtigt werden. Evaluation wird so zum Entwicklungsfaktor (Organisations/Strategieentwicklung, Mittelverteilung) der Hochschule, erhöht die Transparenz der Lehr- und Studienprozesse und stärkt die Eigenverantwortung der Hochschule.

Wesentliche Managementaufgaben des TQM an der TFH Wildau sind das Erfassen der Zufriedenheit der Studenten und Mitarbeiter (Dozenten, Professoren) und das Ableiten und Umsetzen von Maßnahmen. [3]

\subsubsection{Studentische Evaluierung}

Die Evaluierung der Lehre an der TFH Wildau ist eine Umfrage zu einzelnen Vorlesungen, die jeweils gegen Ende der Vorlesungszeit mit Hilfe von Fragebögen durchgeführt wird. Bei der studentischen Evaluierung von Lehrveranstaltungen geht es darum festzustellen, ob der Dozent seine Ziele sinnvoll gewählt hat und ob die Methoden des Dozenten dazu geeignet sind, diese Ziele zu erreichen. Oberstes Ziel der Evaluierung ist die Verbesserung der Qualität der Lehre, darunter werden mehrere Zielsetzungen verfolgt:

1. ein zeitnahes Feedback der Studierenden an die Dozenten

2. Analyse der Lehr- und Lernsituation in den Studiengängen

3. Erkennen von Potenzialen für Verbesserungen der Lehrund Lernsituation

4. Vergleich der Evaluationsergebnisse für Studiengänge über mehrere Semester

5. Umsetzen von geeigneten Maßnahmen in den Fachbereichen und an der Hochschule.

Die Studierenden sind die Zielgruppe der Lehrveranstaltungen. Zufriedene Studenten werden durch hohe Lehrqualität, die eine regelmäßige Kontrolle und stetige Verbesserung erfordert, gewonnen. [3]

\section{Ausgangssituation und Anforderung}

\subsection{Klassische Durchführung der studentischen Lehrevaluation an der TFH Wildau}

Mit Hilfe eines klassischen Erhebungsbogens wurden erstmals im Sommersemester 2003 Meinungen der Studierenden, studienfachgebunden in der letzten Lehrveranstaltung des Semesters, erhoben. Grundlage der Durchführung waren die Verteilerlisten für die einzelnen Fachbereiche. Auf deren Basis wurde ein entsprechender Druckauftrag sowie ein Beschaffungsauftrag für Materialien erteilt. Das umfangreiche Sortieren, Klammern, Eintüten der Erhebungsbögen sowie deren Beschriftung auf Basis der Verteilerlisten erfolgte durch die Autoren des TQM. Diese 
Bögen wurden in den jeweiligen Dekanaten zur Abholung durch die Dozenten bereitgestellt. Diese Planung und Durchführung vollzog sich innerhalb eines Semesters.

Nach Ablauf des Erhebungszeitraumes wurde anhand der Verteilerliste der Rücklauf überprüft. Die Bögen wurden eingelesen und es erfolgte die Erstellung der fachbereichsspezifischen Auswertungsunterlagen. [3]

\subsubsection{Schwächen}

1 Die Planung, Durchführung und Auswertung der schriftlichen Lehrevaluation bedarf eines sehr hohen Kapazitätsbedarfs (Material und Papier)

2 Manuelles Papierhandling und Archivierung erfordert zusätzliche Ressourcen und ist fehleranfällig.

3 Aufgrund des vom Direktstudium abweichenden Studienablaufs und des daraus resultierenden erhöhten Kapazitätsaufwandes ist die Durchführung der Evaluation des Fernstudiums verschoben worden. $\mathrm{Zu}$ dem bestehenden Aufwand der Fernevaluierung führte die nachträglich organisierte Evaluierung und Auswertung zu einem erheblichen Mehraufwand.

4 Durch den festgelegten terminlichen Ablauf konnten dual-orientierte Studiengänge nicht evaluiert werden. Die Evaluierung wurde durch die Studiengangkoordinatoren unabhängig organisiert. Die Ergebnisse waren daher nicht Bestandteil der gesamten Evaluation. Die Erhebungsbögen mussten separat von den übrigen statistisch ausgewertet werden. Insgesamt hatte dies einen beträchtlichen Personalmehraufwand zur Folge.

5 Seminargruppen, welche sich in der Diplomphase befanden, wurden ebenfalls zum genannten Zeitpunkt nicht erfasst. Hier vollzog sich die Handhabung wie unter Punkt (3).

6 Das Programm zur statistischen Auswertung ist bezüglich der Auswahl spezifischer Auswertungsparameter sehr unflexibel.

7 Eingetütete Erhebungsbögen wurden z.T. nicht selbständig durch die Dozenten im Dekanat abgeholt, dadurch entstand für die Mitarbeiter in den Fachbereichsdekanaten ein entsprechender Mehraufwand.

\subsection{Bedarf}

Um diesen Schwächen entgegenzuwirken, wurde die Anwendung der Methode „Online-Befragung“ anstelle der schriftlichen Befragung vom TQM Team analysiert und die Anforderung dem Studiengang Telematik der TFH Wildau übermittelt.

Das Hauptanliegen war, eine höhere Effektivität der studentischen Evaluation zu erreichen. Da die Evaluation begleitend zu allen anderen Aufgaben durchgeführt wird, sollte der Aufwand für die Vorbereitung, Durchführung und Auswertung deutlich reduziert werden und die Auswertung zeitnah den Dozenten zur Verfügung gestellt werden.

\section{Projektbeschreibung}

Um den Evaluierungsprozess effizienter, flexibler und zeitunabhängiger zu gestalten, wurde das „Webbasierte Evaluationssystem" vom Studiengang Telematik ent- wickelt. Es handelt sich dabei um ein Umfrage-System zur elektronischen und webbasierten Unterstützung der gesamten Lehrevaluation. (Abb. 4.1)

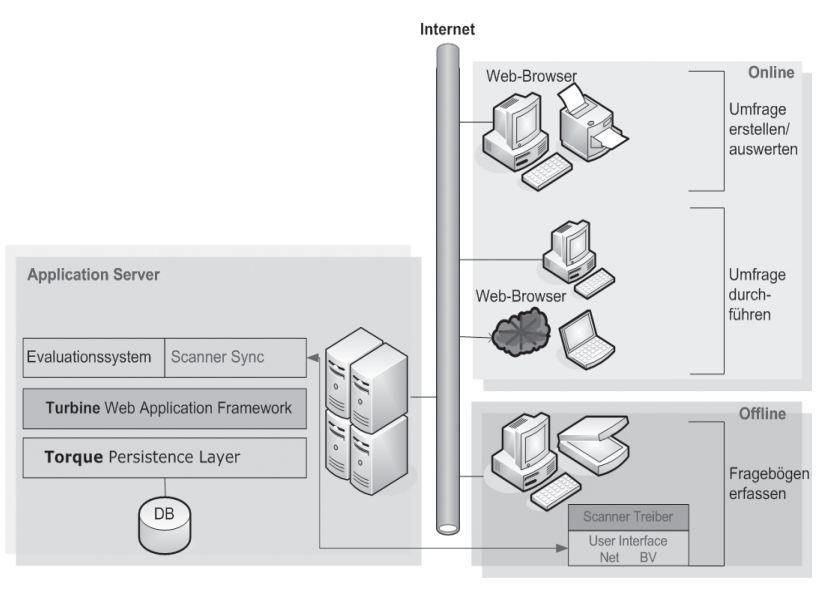

Abb. 4.1: Aufbau des Webbasierten Evaluationssystems

Die wesentlichen Charakteristika sind:

- wahlweise papiergebundenes oder papierloses webbasiertes System,

- anonyme Befragung,

- effiziente und automatisierte Lehrevaluation,

- zeitsparende, einfache Vorbereitung und Auswertung.

Durch unterschiedliche Mechanismen für die Datensicherheit und den Datenschutz kann das webbasierte Evaluierungssystem als $100 \%$ anonym gelten. Es besitzt eine differenzierte Nutzer- und Rollenverwaltung für Administratoren, Dozenten/Mitarbeiter und die (Hochschul-)Leitung. Verschiedene Administratorrollen und mehrstufige Zugriffsrechte erlauben es, intern festzulegen, welche Rolle welche Zugriffsrechte hat (z. B.: Dekan: Einsicht in alle Auswertungen nur der Dozenten seines Fachbereiches durch entsprechendes Ein- bzw. Ausblenden der Dozentennamen).

Unmittelbar nach der Abgabe der Bewertungen durch die Studierenden und Datenerfassung der Fragebögen besteht die Möglichkeit, über passwortgeschützte Dozenten- und Benutzerkonten Einsicht in die Ergebnisse der Evaluation zu nehmen (sofern vom Administrator der entsprechende Zugriff freigegeben wurde).

\subsection{Einführungsphase an der TFH Wildau und erkannte Probleme}

Die Lehrevaluation mit dem webbasierten System wurde vom 03.01. bis 14.01.2005 innerhalb des Direktstudiums Wintersemester 04/05 erstmalig ausschließlich online durchgeführt. Um eine hohe Beteiligung zu erzielen, wurden den Seminargruppen PC-Labore zugeteilt. Im Sommersemester 2005 erfolgte die Evaluation, wiederum ausschließlich online, nach dem Bedarf der Fachbereiche. Eine durchgängige Online-Evaluation in den PC-Laboren der TFH hätte einen ähnlich hohen Aufwand in der Raumplanung wie im WS 04/05 erfordert. Basierend auf den Erfahrungen und Rückmeldungen der Professoren, Lehrbeauftragten und Studenten im WS 04/05 wurden Veränderungen im Ablauf vorgenommen, der Fragebogen überarbeitet und gekürzt sowie das System bezüglich der Einsatzmöglichkeiten weiterentwickelt. 
In diesem Wintersemester wurde die Evaluation in der Zeit vom 12.12.2005 bis 13.01.2006 durchgeführt. Durch die Weiterentwicklung des Evaluierungssystems konnte den Dozenten die Möglichkeit angeboten werden, die Evaluation auch mit vollautomatisch scannbaren Fragebögen durchzuführen (Abb. 4.2). [2]

\begin{tabular}{|c|c|c|c|c|c|c|c|c|c|}
\hline\|\|\|\|\|\|\|\|\|\|\|\|\|\| & \multicolumn{7}{|c|}{ TFH Wildau Evaluationsbogen } & & \\
\hline \multicolumn{10}{|c|}{ Veranstaltung: Telematik (Dr. Michael Abel) Gruppe: Arbeitsfelder } \\
\hline \multicolumn{10}{|c|}{ 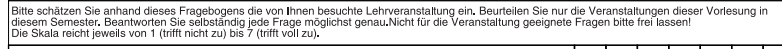 } \\
\hline Fragen: & & & 1 & 2 & 3 & 4 & 5 & te & 7 \\
\hline \multicolumn{9}{|c|}{ Der inhaltiche Autbau der Veranstattung ist logisch/nachvollziehbar } & \\
\hline \multicolumn{9}{|l|}{ Die Veranstaltung ist gut organisiert } & \\
\hline \multicolumn{9}{|c|}{ Der Stoff wird anhand von Beispielen veranschaulicht } & \\
\hline Bedeutung/Relevanz/Nutzen der behand & hen werden nahegelegt & & & & & & & & \\
\hline
\end{tabular}

Abb. 4.2: Ausschnitt des scannbaren Fragebogens mit Barcode

\subsection{Ablauf und Funktionsweise der Lehrevaluation mit dem Evaluierungssystem}

Die Evaluierung der Lehre an der TFH Wildau wird immer gegen Ende der Vorlesungszeit durchgeführt. Folgende Möglichkeiten zur Evaluierung bestehen:

- die Bewertung während der Lehrveranstaltung am PC mit Code

- die Bewertung während der Lehrveranstaltung mit Fragebögen

- die Bewertung außerhalb der Lehrveranstaltung am PC mit Code.

Die ausschließlich webbasierte Befragung bietet sich besonders für Veranstaltungen im EDV-Bereich an. Über das Internet können Teilnehmer mit einem Berechtigungscode Formulare ausfüllen und so papierlos an der Befragung teilnehmen. Zudem kann bequem von zu Hause aus der Online-Fragebogen ausgefüllt werden. (Abb. 4.3)

Der Vorteil der papiergebundenen Umfrage ist die Unabhängigkeit von EDV-Infrastruktur und der dadurch mögliche universelle Einsatz in allen Fachbereichen der Hochschule (z.B. in beliebigen Seminarräumen). [1] [2]

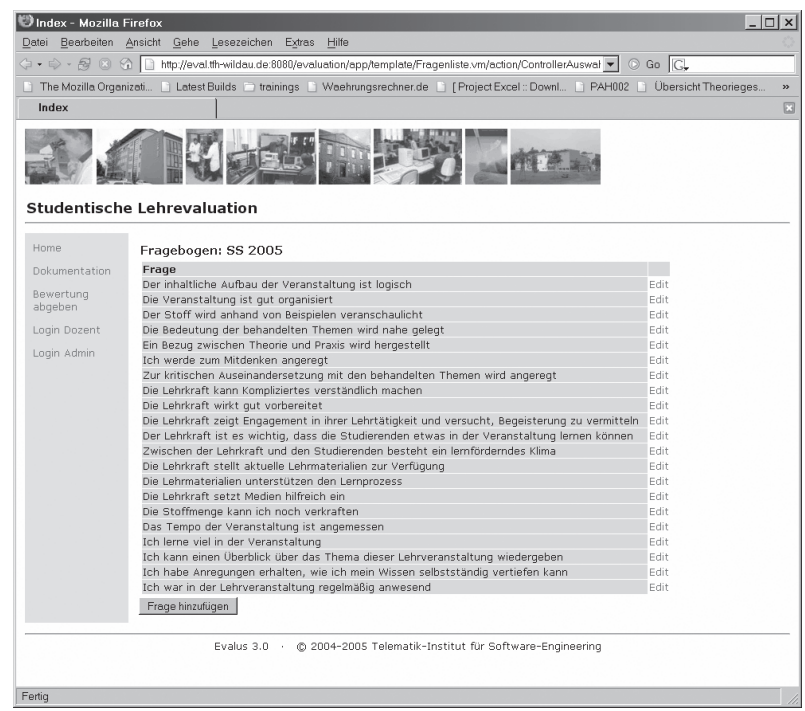

Abb. 4.3: Screenshot: Editierbarer Online-Fragebogen

\subsubsection{Erstellen der Codes/Fragebögen}

Ein Fachbereichs- oder Studiengangsadministrator kann Lehrveranstaltungen beim System anmelden, wobei er nur den Namen der Lehrveranstaltung, die Studiengruppe, die Lehrkraft und die Zahl der Teilnehmer angeben muss. Das System generiert daraufhin für jede Lehrveranstaltung einmal verwendbare Teilnehmercodes in druckbarer Form bzw. Fragebögen, die mit einem Barcode gekennzeichnet sind. Die Anzahl der Codes/Fragebögen entspricht der eingegebenen Seminargruppenstärke.

Die Seminargruppensprecher bzw. die Professoren erhalten nach dem Ausdruck die Codes/Fragebögen in verschlossenen Umschlägen und diese Codes werden im Losverfahren an die Teilnehmer der Lehrveranstaltung verteilt. Die Studierenden loggen sich mit ihrem Zugangscode ein und können den Fragebogen anschließend entweder im Rechnerlabor oder von irgendeinem anderen internetfähigen PC aus (z. B. auch ungestört zu Hause) bearbeiten und abschicken. Die Fragebögen werden ebenfalls in der Lehrveranstaltung verteilt, von den Studenten ausgefüllt, eingesammelt und in einem verschlossenen Umschlag an das TQM zurückgegeben. Die ausgefüllten Fragebögen werden mit Scannern und einer in das System integrierten Bildverarbeitungsapplikation erfasst und von dieser automatisch in die zentrale Datenbank eingepflegt. Jeder Code bzw. Fragebogen kann nur einmal verwendet werden. Dadurch wird sichergestellt, dass Studierende keine Mehrfachbewertungen abgeben können.

Durch dieses Verfahren ist die Anonymität der Studierenden nicht nur gewährleistet, sie ist durch die beschriebene Vorgehensweise auch transparent. Es gibt zu keinem Zeitpunkt eine Verknüpfung zwischen dem Zugangscode und persönlichen Informationen (wie z.B. Email-Adresse, Matrikelnummer), die Studierende bei der Abgabe ihrer Bewertung verunsichern könnte. [1] [2]

\subsubsection{Auswertung}

Sofern vom Hochschul-Administrator der entsprechende Zugriff freigegeben wurde, können Dozenten, Dekane und die Hochschulleitung gemäß ihren Berechtigungen unmittelbar nach Abgabe der Bewertungen online Einsicht in die Auswertung nehmen. Dafür stehen drei verschiedene Auswertungsvarianten zur Verfügung (die jedoch vom Hochschul-Administrator ganz oder teilweise vorübergehend deaktiviert werden können, z.B. während einer Prüfungsphase):

1 Auswertung einer Lehrveranstaltung: zeigt zu jeder Einzelaussage des Fragebogens die Häufigkeiten der einzelnen Antworten (1 bis 7) an. (Abb. 4.4)

2 Gesamtbewertung eines Dozenten: zeigt ebenfalls die Liste aller Aussagen des Fragebogens, jedoch gehen hier nicht die Bewertungen nur einer Lehrveranstaltung, sondern aller Lehrveranstaltungen dieses Dozenten (im jeweiligen Semester) ein. (Abb. 4.5)

3 Dozentenvergleich: erzeugt eine Vergleichsliste entweder aller Lehrkräfte eines Fachbereichs oder der gesamten Hochschule, die entweder alphabetisch sortiert (Abb. 4.6) oder als Rangliste entsprechend dem Bewertungsergebnis ausgegeben werden kann.

Für jeden Dozenten wird zu jeder Frage der Median sowie das Quantil als Referenzparameter ermittelt und farblich dargestellt. Für jeden Studiengang und Fachbereich werden außerdem pro Frage die Mediane über alle Lehrveranstaltungen der Einheit errechnet, um einen Vergleich zu ermöglichen. [1] [2] 


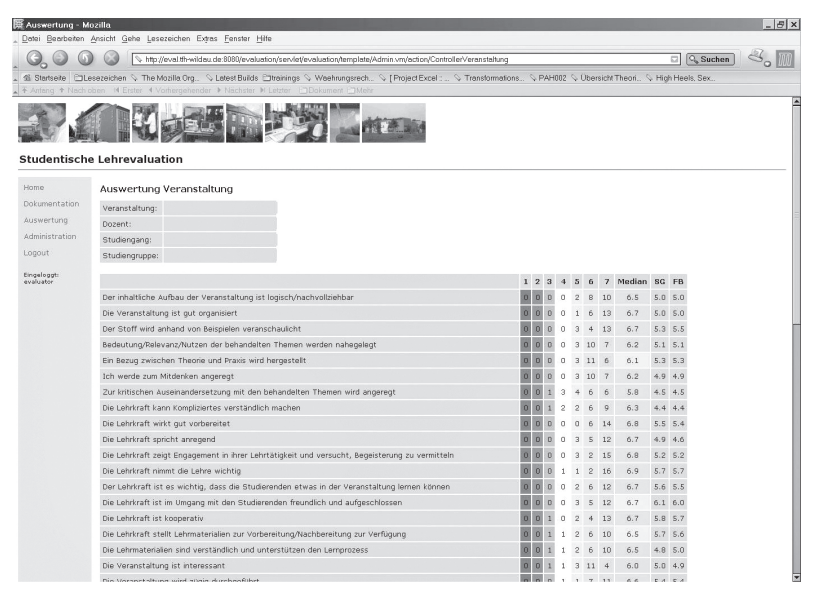

Abb. 4.4: Screen Shot, Auswertung: Lehrveranstaltung

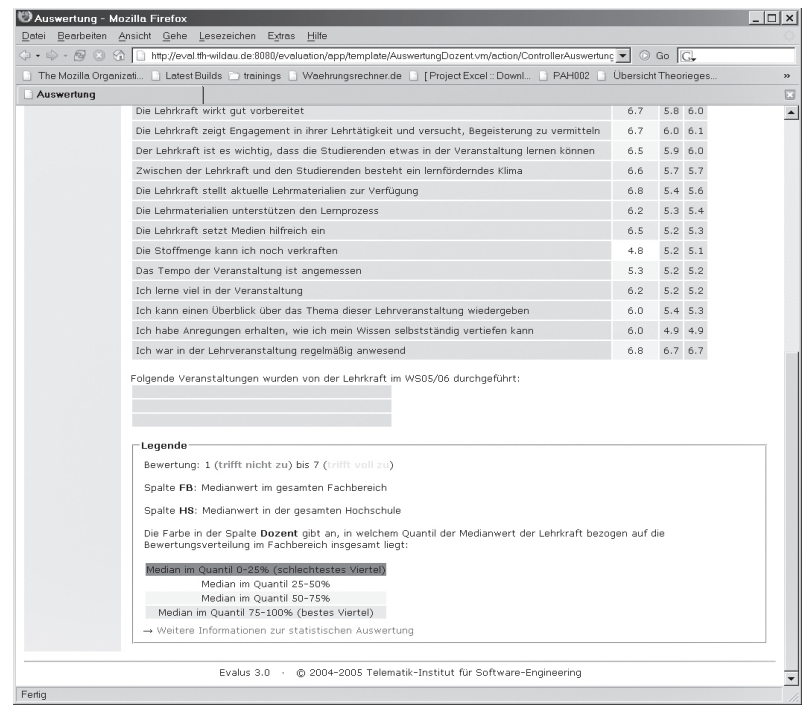

Abb. 4.5: Screen Shot, Auswertung: Gesamtbewertung eines Dozenten

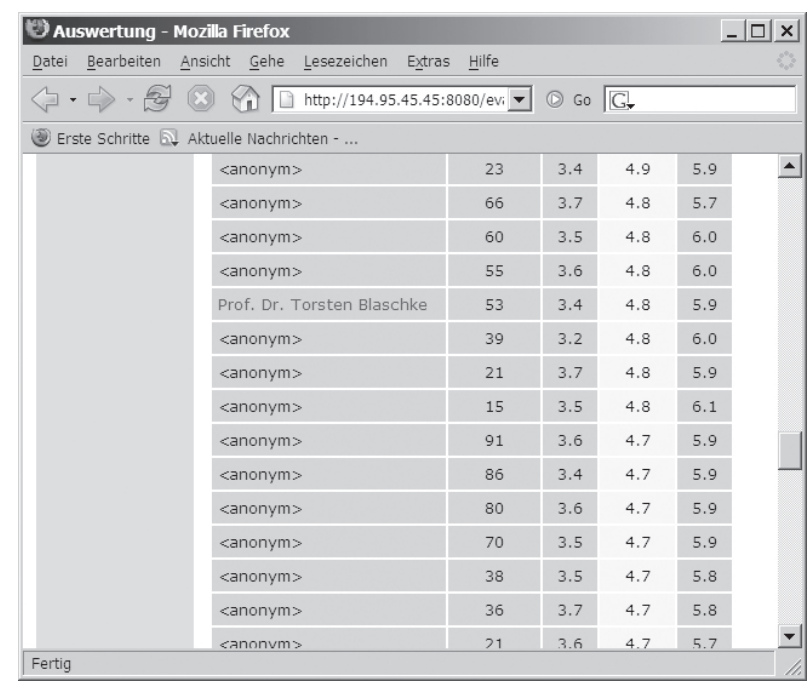

Abb. 4.6: Screen Shot, Auswertung: anonymer Dozentenvergleich

\subsection{Datensicherheit und Datenschutz}

Das webbasierte Evaluationssystem ist mit unterschiedlichen Mechanismen für die Datensicherheit, insbesondere einem Zugriffsschutz ausgestattet. Ein durchdachtes Rechte- und Rollenmodell für Administratoren, Personen der Hochschulleitung und Dozenten sowie die sichere Aufbewahrung der Umfragedaten gewährleisten den Schutz vor Missbrauch.

\subsubsection{Datensicherheit}

Das System hält sämtliche Daten in einer leistungsfähigen Datenbank, so dass außerhalb dieser Datenbank zu keinem Zeitpunkt evaluationsrelevante Daten gehalten werden, auch keine Auswertungs- oder Zwischenergebnisse. Der Hauptgrund dafür ist eine sichere und konsistente Datenhaltung. Durch Integration des Datenbankservers in den Backup-Prozess des Rechenzentrums wird die Datensicherheit gewährleistet.

Das System legt in der Datenbank keine Auswertungsdaten $\mathrm{ab}$, sondern ausschließlich die Original-Bewertungen der Studierenden. Auswertungen werden daraus zur Laufzeit (on-the-fly) generiert, wenn sie angefordert werden.

Sämtliche Bewertungsdaten bleiben in der Datenbank auch nach Ablauf eines Semesters bestehen, so dass Auswertungen noch zu einem viel späteren Zeitpunkt möglich sind, und zwar für alle Nutzergruppen. Damit wird auch die Revisionssicherheit gewährleistet. [1]

\subsubsection{Datenschutz}

Ein weiterer wichtiger Aspekt ist der Datenschutz der Dozenten. Jede Lehrkraft erhält einen eigenen Account für den Zugang zum System, mit dem Auswertungen eigener Lehrveranstaltungen eingesehen werden können, jedoch nicht die anderen Lehrkräfte. Vergleichende Darstellungen werden (soweit überhaupt vom Administrator freigeschaltet) anonymisiert, d.h. alle Dozentennamen (außer dem eigenen) werden ausgeblendet.

Das Zugangssystem verwendet eine sichere 128-Bit Passwortverschlüsselung mit Hilfe eines Hash-Verfahrens (Einweg-Verschlüsselung). Auf diese Weise können Passwörter nicht rekonstruiert werden, nicht einmal vom Datenbank-Administrator. Falls eine Lehrkraft ihr Passwort vergisst, muss vom Administrator oder vom System ein neues Passwort generiert werden. Die Standard-Option sieht vor, dass das Passwort vom System zufällig erzeugt und dann automatisch per Email an die Lehrkraft gesendet wird. Lehrkräfte können ihr Passwort jederzeit ändern. [1]

\subsubsection{Nutzer- und Rollenverwaltung für Administra- toren und Dozenten}

Mit der differenzierten Nutzer- und Rollenverwaltung für Administratoren und Dozenten kann ein DozentenAdministrator Accounts für die Dozenten einrichten. Ein Fachbereichs- oder Studiengangsadministrator kann Lehrveranstaltungen beim System anmelden, wobei er nur den Namen der Lehrveranstaltung, die Studiengruppe, die Lehrkraft und die Zahl der Teilnehmer angeben muss (alternativ kann dies auch von einem Hochschul-Administrator zentral für alle Lehrveranstaltungen gemacht werden). [1]

\subsection{Anonymität}

Ein wesentliches Kriterium für die Aussagekraft eines jeden Evaluationsverfahrens ist die Unbefangenheit der teilnehmenden Bewerter (in diesem Fall der Studierenden). Um diese Unbefangenheit zu gewährleisten, ist es erforderlich, dass das Evaluationsverfahren vollständig anonym ist. Anonymität bedeutet hier, dass keine Lehrkraft in der Lage sein darf, herauszufinden, welche 
Bewertung ein einzelner Student abgegeben hat. Dies wird im System dadurch gewährleistet, dass ein Student in der Datenbank nicht namentlich hinterlegt wird, sondern nur als anonymer Datensatz. Selbst der zugehörige Teilnehmercode für den sicheren Zugang zum System wird unmittelbar nach Abgabe der Bewertung aus der Datenbank gelöscht, so dass es nicht einmal bei Kenntnis des von einem Studierenden verwendeten Teilnehmercodes möglich ist, dessen Bewertung zu ermitteln, auch nicht bei vollem Administrator-Zugriff auf die Datenbank. Das System kann deshalb als $100 \%$ anonym gelten.

Darüber hinaus sollte die Anonymität nicht nur sichergestellt, sie sollte für die Bewerter auch transparent sein. Wegen der oben beschriebenen Vorgehensweise wäre das System beispielsweise auch dann anonym, wenn die Teilnehmercodes per Email an die Studierenden verschickt würden, da die Codes ja nach Abgabe der Bewertung aus der Datenbank gelöscht werden. Für die Studierenden wäre diese Anonymität aber nicht transparent. Es würde ein Zusammenhang zwischen ihrem Zugangscode und einer eindeutigen Kennung ihrer Person (nämlich ihrer Email-Adresse) hergestellt. Sie können nur „glauben“, dass niemand diesen Zusammenhang missbraucht, sicher wissen können sie es nicht. Einem Studierenden, der seinen Zugangscode per Email erhält, kann die Anonymität nicht transparent erscheinen, da bereits die Email selbst eine Verbindung zwischen seiner Identität und dem (vermeintlich anonymem) Code darstellt. Zumindest das versendende System muss also die Verbindung kennen. Die einzig wirklich sichere (und psychologisch überzeugende) Variante ist deshalb die, die Studierenden selbst die Zuordnung zwischen sich und den Codes (und zwar unbeaufsichtigt) vornehmen zu lassen, d.h. durch Verlosen der Codes. Aus diesem Grund haben bei diesem System die Studierenden die Anonymisierung selbst in der Hand. [1]

\section{Softwaretechnische Entwicklung, Tech- nisches Umfeld, Tests und Installation}

Der Weg zur Softwarelösung war und ist durch enge Abstimmung mit dem Qualitätsmanagement der TFH Wildau geprägt. Nur so konnte sichergestellt werden, dass eine Anwendung entwickelt wurde, die den Anforderungen gerecht wird und sich in der Praxis bewährt.

Bei der Entwicklung der Internetapplikationen wurde konsequent auf moderne und leistungsfähige Technologien sowie ausgereifte Open Source Frameworks gesetzt. Auf dieser Basis wird eine breite Palette von Systemplattformen und Schnittstellen unterstützt und die bestmögliche Integration der Applikation in bestehende Infrastrukturen und eine nachhaltige Wartbarkeit gewährleistet.

Das webbasierte Evaluationssystem ist modular und wurde in der plattformunabhängigen Sprache "Java" entwickelt. Die Web-Applikation baut auf den Frameworks Turbine und Torque auf und ist auf jedem browserfähigen Rechner bedienbar.

Turbine ist ein servletbasiertes, als Open Source verfügbares MVC-Framework (MVC = Model View Controller) zur Erstellung von leistungsfähigen und robusten Webapplikationen. Die Idee des MVC-Musters ist die
Trennung von Anzeige (View), Daten (Model) und der Steuerung (Controller). Torque ist als Persistenzschicht das Bindeglied der Applikation zum jeweils verwendeten Datenbanksystem.

Die Scanner-Anwendung ist eigenständig und wurde mit SWT (Standard Widget Toolkit) entwickelt. Darin integriert ist die Bildverarbeitung, welche die eingescannten Barcodes und die Kreuze auf den Papierfragebögen automatisch erkennt, auswertet und über eine eigene HTTPSchnittstelle zum Evaluationsserver sendet.

Im Sommersemester 2004 wurde das System im Studiengang Telematik erfolgreich getestet. Hierbei wurden die technischen Kapazitäten der Telematik-Labore genutzt. Im Wintersemester 2004/2005 konnte die Anwendung hochschulweit eingeführt werden. Die Testläufe ergaben keine technischen Probleme, so dass das System am Hochschulrechenzentrum integriert werden konnte und nun dort gehostet und gepflegt wird.

\section{Projektergebnis}

Die Anforderungen an das System und die Auswertung sind mit diesem Projekt vollständig erfüllt worden. Die entwickelte Software versetzt das TQM in die Lage, groß angelegte Evaluationen von Lehrveranstaltungen, Studiengängen und Fachbereichen ohne langwierige Erfassungs- und Auswertungssaufwände und mit einem hohen Automatisierungsgrad abzuwickeln. Gleichzeitig bleibt die Flexibilität gewährleistet, jederzeit neue Fragebögen zu integrieren und außer der Hauptanwendung Lehrevaluation auch andere Befragungsfelder zu bearbeiten. Neben der Onlinebefragung kann es auch für die Verarbeitung von Papierfragebögen eingesetzt werden. Der Personalaufwand reduziert sich auf ein Minimum und die Software erstellt, vollautomatisiert, sofort nach dem Bewerten die statistische Auswertung in übersichtlicher Form für Dozenten und die administrativen Organe.

Zur Etablierung des Systems an der TFH Wildau trugen im Wesentlichen folgende Punkte bei:

- Die Integration des webbasierten Evaluationssystems in den Evaluierungsprozess der Hochschule,

- dessen stetige Weiterentwicklung und

- seine Anpassung an die konkreten Bedürfnisse der Hochschulorganisation.

Dies wirkte sich auch auf die Umfragebeteiligung aus, die von 51,3\% im Wintersemester 2004/05 auf $62 \%$ im Wintersemester 2005/06 anstieg.

\section{Weitere Planung}

Es ist geplant, das System im gesamten internen Hochschulnetzwerk, incl. der angegliederten Institutionen (Weiterbildung, Fernstudium), einzusetzen. Die im Rahmen des TQM durchgeführten Evaluationen (Evaluierungsarten) weisen Entwicklungspotentiale auf, welche den Einsatz und Weiterentwicklung dieses Systems begründen. Bei der traditionellen Dozentenbefragung sowie der Absolventenbefragung treten ähnliche Schwächen und Probleme auf wie in der studentischen Evaluation (siehe Kapitel 3.1.1 Schwächen). 
Aufgrund des sehr hohen Kapazitätsbedarfs bezüglich der Planung, Durchführung und Auswertung der Dozentenbefragung wurde analog zur studentischen Lehrevaluation die Methode „Online-Befragung“ analysiert. Gegenwärtig wird der Einsatz des flexibel (u. a. individuelle Fragebogengestaltung) auf die Bedürfnisse anpassbaren Systems geplant und vorbereitet.

In Bezug auf das Fernstudium an der TFH Wildau wurde der Online-Fragebogen inhaltlich angepasst und zum Wintersemester 2005/2006 den Fernstudierenden angeboten. Nicht nur angesichts der Tatsache, dass Fernstudenten für ihr Studium bezahlen, wird von ihnen eine qualitativ hochwertige Ausbildung erwartet. Sie sind außerdem vielfach Multiplikatoren, deren Meinung über die TFH Wildau und die dort vorgefundene Studienqualität ganz wesentlich das Image der Hochschule in der Gesellschaft prägt. Um diese Qualität zu messen, bietet sich die Online-Befragung mit dem vorgestellten System in idealer Weise an.

\section{Referenzen}

[1] Ralf Vandenhouten, Dokumentation des webbasierten Evaluationssystems, „Evalus“ Version 3.0, 2004-2005 http://eval.tfh-wildau.de:8080/evaluation/

[2] Internetanwendung „Evalus“ Version 3.0, 2004-2005, http://eval.tfh-wildau.de:8080/evaluation/

[3] Andrea Schmid, Ziele und Aufgaben des TQM an der TFH Wildau, http://www.tfh-wildau.de/tqm

[4] Beschluss: 3. Juli 1995, Zur Evaluation im Hochschulbereich unter besonderer Berücksichtigung der Lehre, Entschließung des 176. Plenums vom 3. Juli 1995, http://www.hrk.de

[5] Beschluss: 21./22. Februar 2000, Evaluation der Lehre - Sachstandsbericht mit Handreichungen, vom 190. Plenum am 21./22. Februar 2000 zur Kenntnis genommen, http://www.hrk.de

\section{Autoren}

Prof. Dr. rer. nat. Ralf Vandenhouten

rvandenh@tfh-wildau.de

Miriam Selz, B. Eng.

mselz@tfh-wildau.de

Technische Fachhochschule Wildau

Fachbereich Ingenieurwesen/Wirtschaftsingenieurwesen Studiengang Telematik

Tel. +49 3375 508-359, -616 